\title{
Relativistic theory of transport in a normal/superconducting microconstriction
}

\author{
C M Aerts and P Strange \\ School of Physical Sciences, University of Kent, Canterbury, UK \\ Received 18 January 2008, in final form 7 March 2008 \\ Published 24 April 2008 \\ Online at stacks.iop.org/JPhysCM/20/215225
}

\begin{abstract}
In this paper we develop a relativistic model of a normal-superconductor junction based on the generalized semiconductor model together with the Dirac-Bogoliubov-de Gennes equations to describe the reflection and transmission of particles at an N-S boundary with a barrier potential. The theory is illustrated with a calculation of the $I-V$ curves, the differential conductance and the excess current. As expected, we observe that relativistic effects are very small in superconducting junctions, but their effect is to increase the effective strength of the barrier.
\end{abstract}

\section{Introduction}

The BCS theory of superconductivity is generally accepted to have been one of the most difficult theories in physics. It was over 40 years between the discovery of superconductivity by Onnes [1] and the advent of an acceptable theory [2, 3]. This theory has been written about many times [4-6], and it leads to agreement with experiment for a large number of superconducting properties. A relativistic version of the theory was only forthcoming in 1995 [7].

An interface or junction between two materials is a fundamental system in the theory of transport. The standard approach to the calculation of characteristic curves for junctions is linear response theory, where the current is calculated as a function of applied potential difference. Using a tunnelling term as a perturbation and Fermi's golden rule, the current across a junction between two metals can be written in terms of the tunnelling matrix element, the Fermi-Dirac distribution function and the densities of states in the two metals. If one of the metals is in the superconducting state there is a fundamental change in the current because the relevant quantity in the superconductor becomes the quasiparticle density of states [5] and the superconducting gap $\Delta$ [8] opens up so no current is observable for $e V<\Delta$. While the perturbation theory approach to such problems works well, it is intrinsically approximate because higher order terms exist [9], and it lacks transparency. Other approaches make use of Green functions at various levels of complexity [10-12], but again these methods are rather physically opaque.

When an electron from a normal metal is incident upon an interface with a superconductor, four different processes can occur, normal reflection, normal transmission, Andreev reflection and quasiparticle transmission. Andreev reflection is a process whereby an electron (strictly an electron-like quasiparticle) of energy $E_{\mathrm{e}}<\Delta / 2$ is incident upon the interface (we have placed the zero of energy at the Fermi energy in the middle of the energy gap) and it is reflected as a hole with equivalent energy $E_{\mathrm{h}}=-E_{\mathrm{e}}$.

One of the successes of the BCS theory is the extension to a description of inhomogeneous superconductors via the Bogoliubov-de Gennes formalism [4] and its use to describe a variety of superconducting properties including transport properties of junctions. The basic equations of this formalism are a pair of coupled Schrödinger-like equations that describe paired electron-like and hole-like quasiparticles. A particularly well cited application is the paper of Blonder et al [13], that sets up and solves the BTK model of transport for a simple model of superconducting microconstrictions. The metal is modelled as a zero potential system and the conducting particles are therefore plane waves. In the superconductor the model is again a system with zero external potential, but with a superconducting order parameter and gap function (taken as a constant). The interface itself is described by a $\delta$-function potential. The incident electrons in the normal metal may be reflected as electrons or undergo Andreev scattering [5] and be reflected as holes. These are matched onto the solutions of the Bogoliubov-de Gennes equations at the boundary and currents can be calculated as a function of applied voltage using a simple method based on a Boltzmann equation approach. The characteristic $I-V$ curve can then be written as a function of temperature and $Z$, a parameter that describes the strength of the $\delta$-function barrier. This original paper has been generalized in several directions $[14,15]$ to three dimensions $[16,17]$, and to deal with magnetic metals [18] and has been used to interpret experiment and to determine material parameters many times; a particularly impressive example for $\mathrm{Cu}-\mathrm{Nb}$ point contacts is reported by Blonder and Tinkham [19], but see also [20-22] for example. 


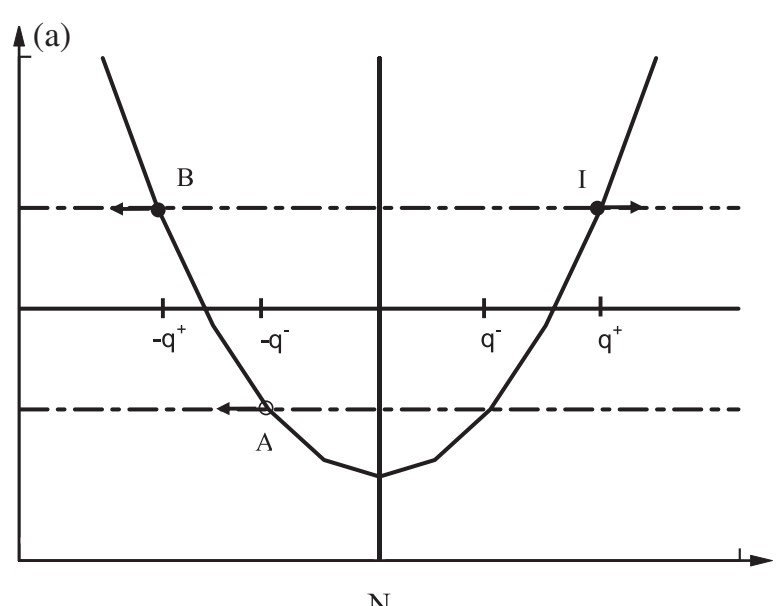

$\mathrm{N}$

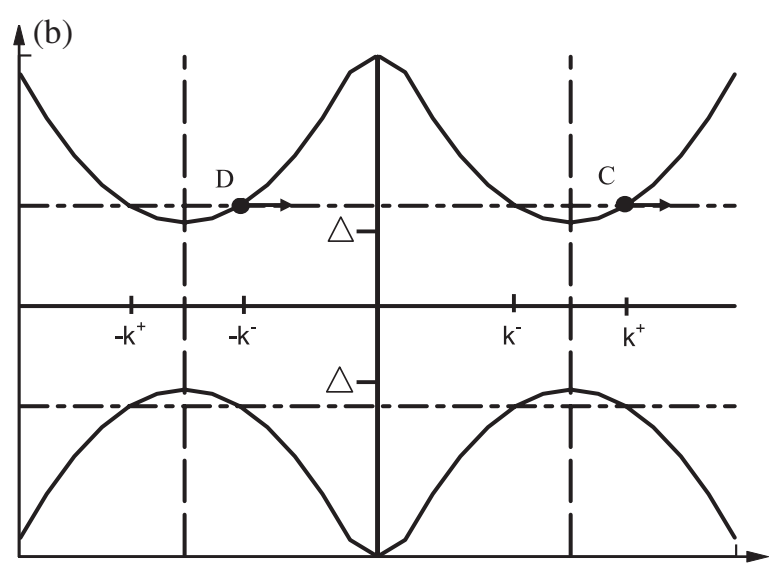

S

Figure 1. Schematic diagram of the energy band structure ( $E$ versus $k$ ) on each side of the interface of an $\mathrm{N}-\mathrm{S}$ junction. The zero of energy is taken to be the chemical potential. (a) The normal metal where the incident electron I with wavevector $q^{+}$may result in a reflected electron B with wavevector $-q^{+}$or hole A with wavevector $-q^{-}$through normal reflection or Andreev scattering, respectively. (b) The superconductor where a particle $(C)$ may emerge from the barrier with a wavevector $\left(k^{+}\right)$that is on the same side of the Fermi surface as the incident particle, or where a particle (D) may emerge with a wavevector $\left(-k^{-}\right)$that is on the opposite side of the Fermi surface from the incident particle.

The purpose of this paper is to set up a relativistic generalization of this theory and to examine any differences between the relativistic and non-relativistic models. In practice, we expect the effects of relativity in BCS superconductors, and in this model for realistic values of the parameters, to be very small. However, we contend that the formalism is itself of interest and how relativity affects the properties of superconductors is of interest even if too small to be directly observable. In this paper we will be concerned with the energy regime close to experiments and will examine the negative energy states within such a model in a future publication.

\section{The model}

We confine our attention to the interface between a normal metal and a superconductor, and by focusing on Andreev reflection we are able to calculate characteristic $I-V$ curves without the additional complication of Josephson effects. We employ the same generalized semiconductor model that was discussed by Blonder et al. In this model the Bogoliubovde Gennes equations are used to model the interface, and this enables us to treat all junctions from a clean interface to one with a strong barrier layer on an equal footing. By matching wavefunctions at the interface we calculate probability currents using fully relativistic quantum theory and use these in the evaluation of the $I-V$ curves. In figure 1 we show the energymomentum relation on both sides of the interface, and this tells us which transitions are allowed energetically. In the normal metal we have an initial electron I with wavevector $q^{+}$and energy $E$ moving in the positive $z$-direction incident upon the interface. $q^{+}$is assumed to be greater then the Fermi wavevector. Two things can happen, either the electron will be reflected as a particle with energy $E$ moving in the negative $z$-direction (B), or Andreev scattering may occur and a hole of energy $-E$ is emitted from the interface (A). If Andreev scattering occurs there must be two extra electron on the superconducting side of the barrier. On the superconducting side we may get an electron with energy $E$ and wavevector $k^{+}$outside the Fermi surface (C) and/or an electron with energy $E$ and wavevector $-k^{-}$inside the Fermi surface (D). As explained by Blonder et al [13], the hole is generated as far below the chemical potential as the electron is above. Once the energetically allowed processes have been identified, the relativistic Bogoliubov-de Gennes equations can be used to determine their probability densities.

\section{Theory}

\subsection{The relativistic Bogoliubov-de Gennes equation}

Capelle and Gross [7] were the first to derive a satisfactory relativistic description of inhomogeneous superconductors. The relativistic generalization of the non-relativistic singlet order parameter [4] is required to be a Kramers pair, and symmetry considerations then tell us it is given by

$$
\Delta(\mathbf{r})=\Psi^{\mathrm{T}}(\mathbf{r}) \eta \Psi(\mathbf{r})
$$

with

$$
\eta=\left(\begin{array}{cccc}
0 & 1 & 0 & 0 \\
-1 & 0 & 0 & 0 \\
0 & 0 & 0 & 1 \\
0 & 0 & -1 & 0
\end{array}\right)
$$

Their theory is then based upon the Hamiltonian

$$
\begin{aligned}
\hat{H}= & \int \mathrm{d}^{3} r \Psi^{\dagger}(\mathbf{r})\left(c \alpha \cdot \hat{\mathbf{p}}+(\beta-I) m c^{2}+e \alpha_{v} A^{v}\right) \Psi(\mathbf{r}) \\
& -\int \mathrm{d}^{3} r\left[\Delta(\mathbf{r}) D^{*}(\mathbf{r})+\text { h.c. }\right]
\end{aligned}
$$

where $\Psi(\mathbf{r})$ represents the four-component Dirac spinor field operator, $D(\mathbf{r})$ is the pair potential, $\alpha$ and $\beta$ are the usual relativistic matrices in the standard representation and

$$
A^{v}=\left(\left(U_{0}(\mathbf{r})-\mu\right) / e, A(\mathbf{r})\right)
$$

with $U_{0}$ the scalar potential, $A(\mathbf{r})$ the usual vector potential and $\mu$ the chemical potential. We have subtracted the electron 
rest mass from the Hamiltonian to facilitate comparison with the non-relativistic limit. The first term in (3) is a single particle Dirac Hamiltonian containing an effective electrostatic potential $U_{0}(\mathbf{r})$, which in principle consists of the Coulomb potential of the lattice and any external potential difference applied to the system. For the purposes of this paper we will assume no magnetic fields are present. The second term contains the coupling of the pair potential to the superconducting order parameter.

The derivation of the relativistic Bogoliubov-de Gennes equation proceeds by application of a relativistic generalization of the Bogoliubov-Valatin [23, 24] transformation.

$$
\Psi_{i}(\mathbf{r})=\sum_{k j}\left[u_{i k j} \gamma_{k j}+v_{i k j}^{*}(\mathbf{r}) \gamma_{k j}^{\dagger}\right] \text {. }
$$

The operators $\gamma_{k j}^{\dagger}$ and $\gamma_{k j}$ are creation and annihilation operators for the new quasiparticles. The index $k$ represents the quantum numbers while $i$ and $j$ refer to the four components of the spinors. Interestingly, in principle this transformation (5) combines elements of the spinors associated with both spins and with particle and antiparticle states. This transformation diagonalizes the Hamiltonian and leads to a set of coupled differential equations, which can be written succinctly as

$$
\left(\begin{array}{cc}
\hat{H}_{\mathrm{D}} & \Delta(\mathbf{r}) \\
\Delta^{\dagger}(\mathbf{r}) & -\hat{H}_{\mathrm{D}}^{*}
\end{array}\right)\left(\begin{array}{c}
u(\mathbf{r}, t) \\
v(\mathbf{r}, t)
\end{array}\right)=\mathrm{i} \hbar \frac{\partial}{\partial t}\left(\begin{array}{c}
u(\mathbf{r}, t) \\
v(\mathbf{r}, t)
\end{array}\right) .
$$

This is the Dirac-Bogoliubov-de Gennes equation. It is an $8 \times$ 8 equation; $u(\mathbf{r}, t)$ and $v(\mathbf{r}, t)$ are four component quantities. $\hat{H}_{\mathrm{D}}$ is the standard single particle Dirac Hamiltonian [25]

$$
\hat{H}_{\mathrm{D}}=c \alpha \cdot \hat{p}+(\beta-I) m c^{2}+U_{0}(\mathbf{r})-\mu .
$$

One key element of this analysis is the position of the zero of energy. Equation (7) sets the zero of energy at the chemical potential. This means that energies below the Fermi energy are negative. Negative energies in this context should not be confused with the negative energies usually discussed in relativistic quantum theory, which have a correspondence to antiparticles.

Before solving these equations generally, consider what happens as $\Delta \rightarrow 0$. In this case the equations separate into two $4 \times 4$ Dirac equations, one of which describes an electron above the Fermi energy. The Dirac equation in $v(\mathbf{r}, t)$ requires slightly more interpretation. Acting upon it with the time-reversal operator yields the Dirac equation at negative energies, so $v(\mathbf{r}, t)$ represents a time-reversed electron below the Fermi energy.

To solve equations (6) we will follow the spirit of Blonder et al [13] and set $\mu$ and $U(\mathbf{r})$ constant and let $\Delta(\mathbf{r}) \rightarrow \Delta \eta$ with $\Delta$ constant. We will also limit ourselves to one dimension $(z)$ and then

$$
\begin{aligned}
& u(\mathbf{r}, t) \rightarrow u(z, t)=\left(\begin{array}{l}
u_{1} \\
u_{2} \\
u_{3} \\
u_{4}
\end{array}\right) \mathrm{e}^{\mathrm{i}(p z-E t) / \hbar}=u(z) \mathrm{e}^{\mathrm{i}(p z-E t) / \hbar} \\
& v(\mathbf{r}, t) \rightarrow v(z, t)=\left(\begin{array}{l}
v_{1} \\
v_{2} \\
v_{3} \\
v_{4}
\end{array}\right) \mathrm{e}^{\mathrm{i}(p z-E t) / \hbar}=v(z) \mathrm{e}^{\mathrm{i}(p z-E t) / \hbar}
\end{aligned}
$$

where we have assumed that the elements in the column matrices are independent of both $z$ and time. It is trivial to generalize the non-relativistic interpretation of $u$ and $v$ [6]. $\left|u_{1(2)}\right|^{2}$ is the probability that the quasiparticle is a spin up (down) electron and $\left|v_{1(2)}\right|^{2}$ is the probability that the quasiparticle is a spin up (down) hole. Putting equations (8) and (9) into equation (6), remembering that $\alpha_{z}=\alpha_{z}^{*}, \hat{p}_{z}^{*}=$ $-\hat{p}_{z}$ and insisting that $\Delta$ is real we obtain

$$
\left(c \alpha_{z} p_{z}+(\beta-I) m c^{2}+U_{0}-\mu\right) u(z)+\Delta \eta v(z)=E u(z, t)
$$

$$
\begin{aligned}
& -\left(-c \alpha_{z} p_{z}+(\beta-I) m c^{2}+U_{0}-\mu\right) v(z) \\
& -\Delta \eta u(z)=E v(z, t) .
\end{aligned}
$$

Now we will follow Capelle and Gross [7] and set $U_{0}=0$ and note that $\Delta$ is very small. Therefore, it is still a very good approximation to write

$$
\left(c \alpha_{z} p_{z}+(\beta-I) m c^{2}\right) u(z)=\left(W-m c^{2}\right) u(z)
$$

where $W$ is the energy of a relativistic free particle. $W$ is independent of the direction of travel and so we can simplify equations (10) and (11),

$$
\begin{gathered}
\left(W-m c^{2}-\mu\right) u(z)+\Delta \eta v(z)=E u(z) \\
-\left(W-m c^{2}-\mu\right) v(z)-\Delta \eta u(z)=E v(z) .
\end{gathered}
$$

Equations (13) are actually eight simultaneous algebraic equations in eight unknowns. It is useful to write these out explicitly,

$$
\begin{aligned}
& \left(E-\left(W-m c^{2}-\mu\right)\right) u_{1}-\Delta v_{2}=0 \\
& \left(E-\left(W-m c^{2}-\mu\right)\right) u_{2}+\Delta v_{1}=0 \\
& \left(E-\left(W-m c^{2}-\mu\right)\right) u_{3}-\Delta v_{4}=0 \\
& \left(E-\left(W-m c^{2}-\mu\right)\right) u_{4}+\Delta v_{3}=0 \\
& \left(E+\left(W-m c^{2}-\mu\right)\right) v_{1}+\Delta u_{2}=0 \\
& \left(E+\left(W-m c^{2}-\mu\right)\right) v_{2}-\Delta u_{1}=0 \\
& \left(E+\left(W-m c^{2}-\mu\right)\right) v_{3}+\Delta u_{4}=0 \\
& \left(E+\left(W-m c^{2}-\mu\right)\right) v_{4}-\Delta u_{3}=0 .
\end{aligned}
$$

It is clear that the eight equations have separated into four sets of two equations, which are now trivial to solve. This separation was facilitated by choosing $z$ as the direction of motion. Each pair of equations has a non-trivial solution if

$$
E(k)=\sqrt{\left(W(k)-m c^{2}-\mu\right)^{2}+\Delta^{2}}
$$

with

$$
W(k)= \pm \sqrt{\left(c^{2} \hbar^{2} k^{2}+m^{2} c^{4}\right)}
$$

where we have explicitly indicated the dependence of the energy on wavevector. This energy spectrum has four branches corresponding to the four possible choices of sign. Equation (15) has the correct limits as $\Delta \rightarrow 0$, and as $v / c \rightarrow 0$ it becomes the well known equation of BCS theory $E_{k}= \pm \sqrt{\hbar^{2} k^{2} / 2 m+|\Delta|^{2}}$. Clearly from equations (14) we 
can set six of $u_{1} \cdots u_{4}$ and $v_{1} \cdots v_{4}$ equal to zero and solve the Bogoliubov-de Gennes equations for just two non-zero components of the wavefunction. We will also insist on the normalization

$$
\sum_{j=1}^{4}\left(\left|u_{j}\right|^{2}+\left|v_{j}\right|^{2}\right)=1 .
$$

Equations (14) are purely real and $u_{j}$ and $v_{j}$ can be chosen to be real when (15) is satisfied. This will always be true when the solution to the Bogoliubov-de Gennes equation is wavelike. If we choose $u_{1}$ and $v_{2}$ as the non-zero parts of the wavefunction and take the positive square root in equation (15) we then find

$$
\begin{aligned}
& u_{1}^{2}=\frac{1}{2}\left(1+\frac{\left(E^{2}-\Delta^{2}\right)^{1 / 2}}{E}\right) \\
& v_{2}^{2}=\frac{1}{2}\left(1-\frac{\left(E^{2}-\Delta^{2}\right)^{1 / 2}}{E}\right)
\end{aligned}
$$

and we obtain identical expressions if we choose the pairs $\left(u_{2}, v_{1}\right),\left(u_{3}, v_{4}\right)$ or $\left(u_{4}, v_{3}\right)$ to be the non-zero components. If we had taken the negative square root in equation (15) the expressions would have looked very similar; the only change would have been that the signs after the 1 in the brackets would have swapped between the $u_{1}$ and the $v_{2}$ equations. For a given energy there are two allowed values of the wavevector

$$
k^{ \pm}=\frac{1}{c \hbar}\left(\left(\mu+m c^{2} \pm\left(E^{2}-\Delta^{2}\right)^{1 / 2}\right)^{2}-m^{2} c^{4}\right)^{1 / 2} .
$$

So, our solutions to the Bogoliubov-de Gennes equations are

$$
\begin{aligned}
& \psi(\mathbf{r}, t)=\left(\begin{array}{c}
u(\mathbf{r}, t) \\
v(\mathbf{r}, t)
\end{array}\right)=\left(\begin{array}{c}
u_{1} \\
0 \\
0 \\
0 \\
0 \\
v_{2} \\
0 \\
0
\end{array}\right) \mathrm{e}^{ \pm \mathrm{i}\left(p_{z}^{+} z-E t\right) / \hbar} \\
& \psi(\mathbf{r}, t)=\left(\begin{array}{c}
u(\mathbf{r}, t) \\
v(\mathbf{r}, t)
\end{array}\right)=\left(\begin{array}{c}
v_{2} \\
0 \\
0 \\
0 \\
0 \\
u_{1} \\
0 \\
0
\end{array}\right) \mathrm{e}^{ \pm \mathrm{i}\left(p_{z}^{-} z-E t\right) / \hbar}
\end{aligned}
$$

and similarly for the $\left(u_{2}, v_{1}\right),\left(u_{3}, v_{4}\right)$ and $\left(u_{4}, v_{3}\right)$ combinations, and a general solution is a linear combination of these four independent sets of solutions.

While equations (20) represent the general solution of the Bogoliubov-de Gennes equations under the given approximations, they do not satisfy the boundary condition that they must become solutions of the usual free particle Dirac equation when $\Delta \rightarrow 0$. To satisfy this condition we require the solutions to the free particle Dirac equation. These have been evaluated many times [25] and we just quote the results here. For particles free to move in the positive $z$-direction

$$
\begin{aligned}
& \psi_{1}(z)=\frac{1}{\sqrt{V}}\left(\frac{W+m c^{2}}{W}\right)^{1 / 2}\left(\begin{array}{c}
1 \\
0 \\
\frac{c \hbar k}{W+m c^{2}} \\
0
\end{array}\right) \mathrm{e}^{\mathrm{i}\left(p_{z} z-W t\right) / \hbar} \\
& \psi_{2}(z)=\frac{1}{\sqrt{V}}\left(\frac{W+m c^{2}}{W}\right)^{1 / 2}\left(\begin{array}{c}
0 \\
1 \\
0 \\
-\frac{c \hbar k}{W+m c^{2}}
\end{array}\right) \mathrm{e}^{\mathrm{i}\left(p_{z} z-W t\right) / \hbar} \\
& \psi_{3}(z)=\frac{1}{\sqrt{V}}\left(\frac{W+m c^{2}}{W}\right)^{1 / 2}\left(\begin{array}{c}
\frac{c \hbar k}{W+m c^{2}} \\
0 \\
1 \\
0
\end{array}\right) \mathrm{e}^{\mathrm{i}\left(p_{z} z+W t\right) / \hbar} \\
& \psi_{4}(z)=\frac{1}{\sqrt{V}}\left(\frac{W+m c^{2}}{W}\right)^{1 / 2}\left(\begin{array}{c}
0 \\
-\frac{c \hbar k}{W+m c^{2}} \\
0 \\
1
\end{array}\right) \mathrm{e}^{\mathrm{i}\left(p_{z} z+W t\right) / \hbar}
\end{aligned}
$$

corresponding to a spin up positive energy particle, a spin down positive energy particle, a spin up negative energy (in this case we mean negative energy in the sense of corresponding to antiparticle states) particle and a spin down negative energy particle respectively. Here $V$ is the normalization volume. For particles travelling in the negative $z$-direction we just change the sign of $k$ in these expressions. In the columns in $\psi_{n}(x)$ the unity element is called the large component of the wavefunction and the other non-zero component is the small component.

Now solutions of the form (20) must map onto solutions (21) when $\Delta \rightarrow 0$. Equation (20) represents a spin up particle coupled to a spin down hole. It has only two non-zero components whereas it should have four for these solutions to map onto (21). Insisting that this limit is obeyed brings about a considerable simplification.

$$
\begin{aligned}
u_{3} & =\frac{c p_{z}}{W+m c^{2}} u_{1} & u_{4} & =-\frac{c p_{z}}{W+m c^{2}} u_{2} \\
v_{3} & =-\frac{c_{p} z}{W+m c^{2}} v_{1} & v_{4} & =\frac{c p_{z}}{W+m c^{2}} v_{2}
\end{aligned}
$$

and the final solutions are

$$
\begin{gathered}
\psi(\mathbf{r}, t)=c\left(\begin{array}{c}
u_{1} \\
0 \\
\frac{c p_{z}}{W+m c^{2}} u_{1} \\
0 \\
0 \\
v_{2} \\
0 \\
\frac{c p_{z}}{W+m c^{2}} v_{2}
\end{array}\right) \mathrm{e}^{\mathrm{i}\left(p_{z}^{+} z-E t\right) / \hbar} \\
+d\left(\begin{array}{c}
v_{2} \\
0 \\
-\frac{c p_{z}}{W+m c^{2}} v_{2} \\
0 \\
0 \\
u_{1} \\
0 \\
-\frac{c p_{z}}{W+m c^{2}} u_{1}
\end{array}\right) \mathrm{e}^{-\mathrm{i}\left(p_{z}^{-} z-E t\right) / \hbar}
\end{gathered}
$$


for a quasi-particle composed of a spin up electron and a spin down hole if there is no branch crossing or a spin up hole and a spin down electron if there is branch crossing.

$\psi(\mathbf{r}, t)=e\left(\begin{array}{c}0 \\ u_{2} \\ 0 \\ -\frac{c p_{z}}{W+m c^{2}} u_{2} v_{1} \\ 0 \\ -\frac{c p_{z}}{W+m c^{2}} v_{1} \\ 0\end{array}\right) \mathrm{e}^{\mathrm{i}\left(p_{z}^{+} z-E t\right) / \hbar}$

$$
+f\left(\begin{array}{c}
0 \\
v_{1} \\
0 \\
\frac{c p_{z}}{W+m c^{2}} v_{1} \\
u_{2} \\
0 \\
\frac{c p_{z}}{W+m c^{2}} u_{2} \\
0
\end{array}\right) \mathrm{e}^{-\mathrm{i}\left(p_{z}^{-} z-E t\right) / \hbar}
$$

for a quasiparticle composed of a spin down electron and a spin up hole if there is no branch crossing or a spin down hole and a spin up electron if there is branch crossing. There should be no confusion between the coefficient $c$ in equation (23) and the speed of light $c$.

\subsection{The normal/superconductor junction}

We are now in a position to set up a model of a normal metal/superconductor junction. The normal metal is described in the free electron approximation and the superconductor as the free solutions of the Bogoliubov-de Gennes equations described above. The boundary will be described by a deltafunction potential, i.e. $V(z)=H \delta(z) ; H$ is an adjustable parameter representing the strength of the barrier. Rigorously, a $\delta$-function potential leads to considerable difficulties in relativistic quantum theory. Because it is higher than $2 m c^{2}$ it can lead to the Klein paradox, and, as discussed by Fairbairn [26] among others in the context of the KronigPenney model, the $\delta$-function can be written as a mathematical limit in several different ways and the physics of the model is determined by how that limit is taken. If the $\delta$-function is described as some limit of a potential barrier as the height becomes infinite and the width infinitesimal, it becomes necessary to solve the Bogoliubov-de Gennes equations in the barrier, and this adds an extra unnecessary layer of complexity that is contrary to the spirit of the BTK model. We do not expect that particle/antiparticle creation has a significant role to play in the properties of superconducting junctions, as we work at energies much smaller than $m c^{2}$. Therefore, we employ a simple relativistic generalization of the non-relativistic $\delta$ function boundary conditions. This, at least, has the correct non-relativistic limit.

Starting with equations (6) it is straightforward to derive the appropriate boundary conditions that have to be obeyed by steady state plane wave solutions at the $\mathrm{N}-\mathrm{S}$ interface. They are the following.

(1) The large components of the wavefunctions either side of the boundary have to match onto one another.
(2) The small components of the wavefunction are chosen so that the wavefunction at the boundary is a solution of the Bogoliubov-de Gennes equation, i.e.

$$
\begin{gathered}
-\mathrm{i} \hbar c \alpha_{z}\left(u_{S}(0)-u_{N}(0)\right)+H u(0)=0 \\
\mathrm{i} \hbar c \alpha_{z}\left(v_{S}(0)-v_{N}(0)\right)+H v(0)=0
\end{gathered}
$$

where $u_{S(N)}$ are the top four components of the wavefunction on the superconducting (normal) side of the barrier, and $v_{S(N)}$ are the equivalent lower four components.

It will be important to determine the current densities for the various processes that may occur at the superconducting/normal interface. The expression we have to use to calculate this is derived in appendix A. In units of $2 c^{2} p_{\mathrm{F}} /\left(W+m c^{2}\right)$ (which reduces to the Fermi velocity in the non-relativistic limit) we find they are

$$
\begin{gathered}
A(E)=-|a|^{2} \quad B(E)=-|b|^{2} \\
C(E)=\left(\left|u_{1}\right|^{2}-\left|v_{2}\right|^{2}\right)|c|^{2} \\
D(E)=\left(\left|u_{1}\right|^{2}-\left|v_{2}\right|^{2}\right)|d|^{2} \\
H(E)=\left(\left|u_{2}\right|^{2}-\left|v_{1}\right|^{2}\right)|e|^{2} \\
F(E)=\left(\left|u_{2}\right|^{2}-\left|v_{1}\right|^{2}\right)|f|^{2} .
\end{gathered}
$$

Since plane waves are spatially uniform, there is no need to specify position in these definitions. $a$ and $b$ in equations (26) are the coefficients of the Andreev reflected hole and the reflected electron respectively.

\subsection{Scattering at the interface in the BTK approximation}

In this section we report a direct relativistic generalization of the BTK results, following their methods and making equivalent approximations to that paper. While we expect relativistic effects at the normal/superconductor interface to be small at most, the relativistic formalism automatically manifests the effects of spin on the scattering as well. Let us consider a spin up electron incident upon the boundary from the normal metal side. Then there are in principal four possibilities for scattering on the normal side. After scattering we can have on the normal side (i) a spin up electron and a spin down hole, (ii) a spin up electron and a spin up hole, (iii) a spin down electron and a spin up hole, (iv) a spin down electron and a spin down hole. If the incident electron is spin down then all the conclusions of the following sections are still valid, provided we everywhere replace spin up with spin down and vice versa. We now examine each of these possibilities in turn. The wavefunction on the superconducting side of the boundary is given by the sum of equations (23) and (24) (we have dropped the irrelevant time dependence). The normalization has also been dropped, as it would cancel later in the calculation anyway. This is a scattering problem and conventionally we normalize to a unit incident particle. The prefactor $I$ in front of the incident particle is indeed unity unless otherwise stated. 
3.3.1. A spin up electron and a spin up hole. In this case both the incident electron and the scattered hole have parallel spin. The incident and normally reflected electron still have the same spin, i.e. we have not allowed any spin flip at the interface. The particle on the normal side of the interface is given by

$$
\begin{aligned}
& \psi_{n}(z)=I\left(\begin{array}{c}
1 \\
0 \\
\frac{c \hbar k}{W+m c^{2}} \\
0 \\
0 \\
0 \\
0 \\
0
\end{array}\right) \mathrm{e}^{i p_{z} z / \hbar}+a\left(\begin{array}{c}
0 \\
0 \\
0 \\
0 \\
i \\
0 \\
-\frac{i c p_{z}}{W+m c^{2}} \\
0
\end{array}\right) \mathrm{e}^{i p_{z}^{+} z / \hbar} \\
& +b\left(\begin{array}{c}
1 \\
0 \\
-\frac{c \hbar k}{W+m c^{2}} \\
0 \\
0 \\
0 \\
0 \\
0
\end{array}\right) \mathrm{e}^{-i p_{z} z / \hbar}
\end{aligned}
$$

The boundary conditions can then be written down and they are trivial to deal with. It is immediately obvious that

$$
c=d=e=f=a=0
$$

and there is no solution for $b$, which means that this process is not possible.

3.3.2. A spin up electron and a spin down hole. In this instance we take the case where the electron may undergo normal reflection or Andreev scattering as a spin down hole, i.e. the electron does not undergo a spin-flip at the boundary. So, on the normal side of the barrier the electron wavefunction is

$$
\begin{aligned}
& \psi_{n}(z)=I\left(\begin{array}{c}
1 \\
0 \\
\frac{c \hbar k}{W+m c^{2}} \\
0 \\
0 \\
0 \\
0 \\
0
\end{array}\right) \mathrm{e}^{i p_{z} z / \hbar}+a\left(\begin{array}{c}
0 \\
0 \\
0 \\
0 \\
0 \\
i \\
0 \\
\frac{i c p_{z}}{W+m c^{2}}
\end{array}\right) \mathrm{e}^{i p_{z}^{+} z / \hbar} \\
& +b\left(\begin{array}{c}
1 \\
0 \\
-\frac{c \hbar k}{W+m c^{2}} \\
0 \\
0 \\
0 \\
0 \\
0
\end{array}\right) \mathrm{e}^{-i p_{z} z / \hbar}
\end{aligned}
$$

where the $i$ in the Andreev scattering term is a phase factor simply introduced to facilitate some of the arithmetic. There is no loss of generality in including this as it will also be incorporated into $a$.

If $I=1$ the first boundary condition gives us

$$
\begin{gathered}
1+b=c u_{1}+d v_{2} \\
i a=c v_{2}+d u_{1} \\
0=e u_{2}+f v_{1} \\
0=e v_{1}+f u_{2}
\end{gathered}
$$

and the second boundary condition yields

$$
\begin{gathered}
-\frac{i \hbar c^{2} p_{z}}{W+m c^{2}}\left(\left(c u_{1}-d v_{2}\right)-(1-b)\right)+H(1+b)=0 \\
-\frac{i \hbar c^{2} p_{z}}{W+m c^{2}}\left(i a-\left(c v_{2}-d u_{1}\right)\right)-i H a=0 \\
\left(f v_{1}-e u_{2}\right)=0 \\
\left(f u_{2}-e v_{1}\right)=0 .
\end{gathered}
$$

Evidently these immediately give $e=f=0$. Analogously to Blonder et al, we set

$$
Z=\frac{\left(W+m c^{2}\right) H}{2 \hbar c^{2} p_{z}} \approx \frac{\left(W+m c^{2}\right) H}{2 \hbar^{2} c^{2} k_{\mathrm{F}}}
$$

where $k_{\mathrm{F}}$ is the Fermi wavevector and

$$
\gamma=Z^{2}\left(\left|u_{1}\right|^{2}-\left|v_{2}\right|^{2}\right)+\left|u_{1}\right|^{2}
$$

and a little further algebra yields

$$
\begin{gathered}
a=-\frac{i u_{1} v_{2}}{\gamma} \quad b=-\frac{\left(\left|u_{1}\right|^{2}-\left|v_{2}\right|^{2}\right)\left(Z^{2}+i Z\right)}{\gamma} \\
c=\frac{(1-i Z) u_{1}}{\gamma} \quad d=\frac{i Z v_{2}}{\gamma} .
\end{gathered}
$$

These equations look very similar to their non-relativistic equivalents. Following BTK we have set $k^{+}=k^{-}=$ $q^{+}=q^{-}=k_{\mathrm{F}}$, as this should be an excellent approximation and simplifies the mathematics considerably. If we consider the non-relativistic limit of $Z, W \rightarrow m c^{2}$ and

$$
Z \rightarrow \frac{m H}{\hbar p_{\mathrm{F}}}=\frac{H}{\hbar v_{\mathrm{F}}}
$$

where $v_{\mathrm{F}}$ is the Fermi velocity and this equation is identical to the definition of $Z$ given by Blonder et al [13].

We are now in a position to calculate these current densities using the parameters within the model and equations (26). Typical values for these parameters are shown in figure 2 . These are characterized by $H$ rather than the more conventional $Z$ to facilitate comparison with a later section, where $Z$ is an inappropriate parameter. These curves were calculated using the relativistic formalism, but are identical to the non-relativistic curves of BTK for the same value of $H$. The reason for this is clear. Any 

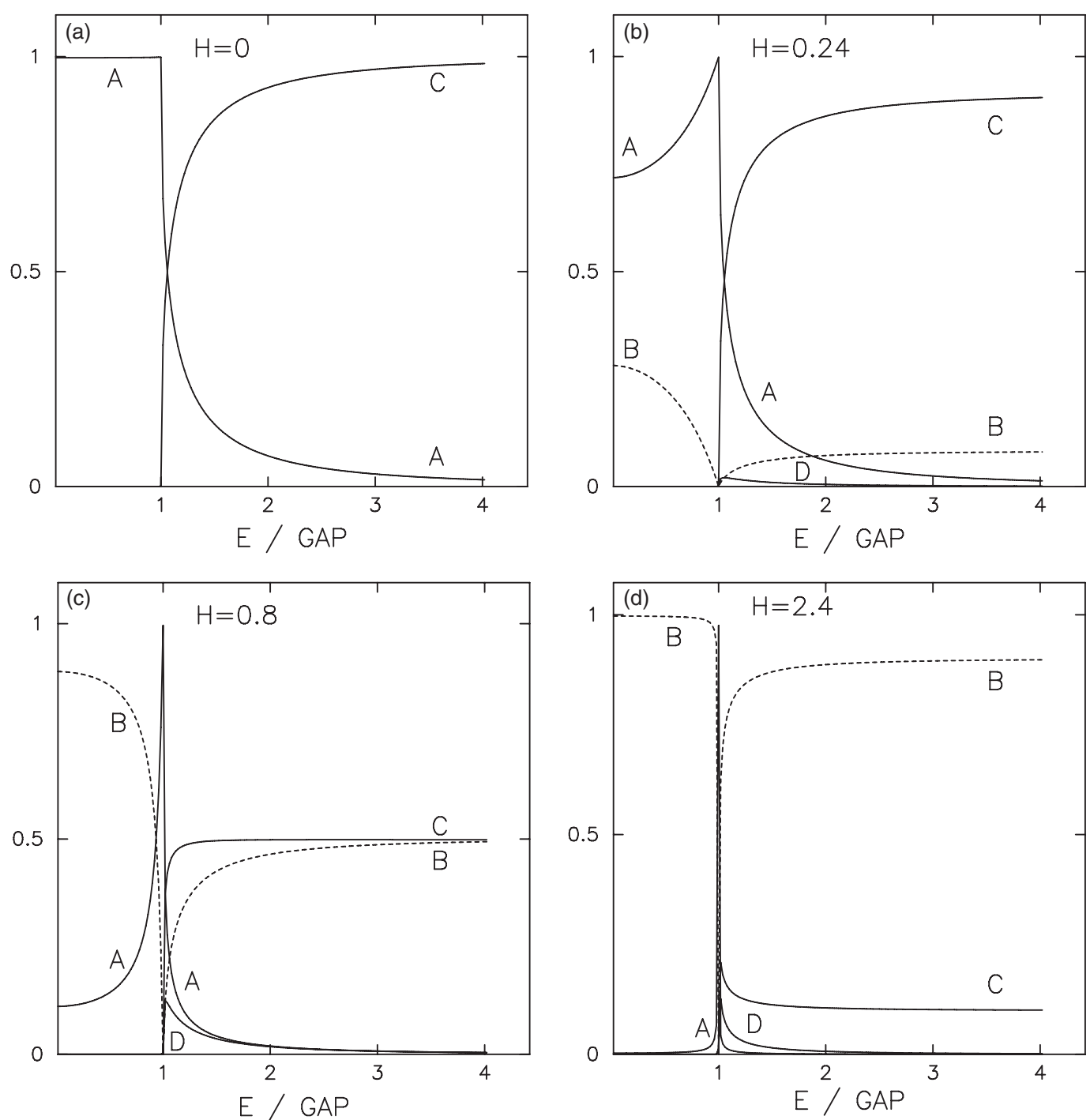

Figure 2. Reflection and transmission coefficients at the N-S interface for a variety of barrier strengths as a function of energy. This is for the case of a spin up electron and a spin down hole on the normal side of the barrier. The quantities plotted are given by equations (26) and (43) and (44). The energy coordinate has been made independent of the size of the model parameters by writing it in units of the energy gap. $A$ is the probability of Andreev reflection, $B$ is the probability of normal reflection, $C$ is the probability of transmission through the interface without a band branch crossing and $D$ is the probability of transmission with a band branch crossing.

problem is defined by the value of two quantities, the gap $\Delta$ and $Z$. Once these have been defined, the solutions for energy $E$ involve equations (18) and (34). These are identical to their non-relativistic counterparts, even though the definition of $E$ differs in the two cases. So we are solving exactly the same equations as the non-relativistic case, so no relativistic effects can be manifest. It is inconceivable that relativity could play no part in the description of the $\mathrm{N}-\mathrm{S}$ junction for some values of the parameters. However, we have made the BTK approximation that $k^{+}=k^{-}=$ $q^{+}=q^{-}=k_{\mathrm{F}}$. Of course, this removes some relative motion from the problem and so removes relativistic effects.

3.3.3. A spin down electron and a spin up hole. In this case the normal state wavefunction is

$$
\begin{aligned}
& \psi_{n}(z)=I\left(\begin{array}{c}
1 \\
0 \\
\frac{c \hbar k}{W+m c^{2}} \\
0 \\
0 \\
0 \\
0 \\
0
\end{array}\right) \mathrm{e}^{i p_{z} z / \hbar}+a\left(\begin{array}{c}
0 \\
0 \\
0 \\
0 \\
i \\
0 \\
0 \\
1 \\
-\frac{i c p_{z}}{W+m c^{2}} \\
0
\end{array}\right) \mathrm{e}^{i p_{z}^{+} z / \hbar} \\
& +b\left(\begin{array}{c}
\frac{c \hbar k}{W+m c^{2}} \\
0 \\
0 \\
0 \\
0
\end{array}\right) \mathrm{e}^{-i p_{z} z / \hbar}
\end{aligned}
$$



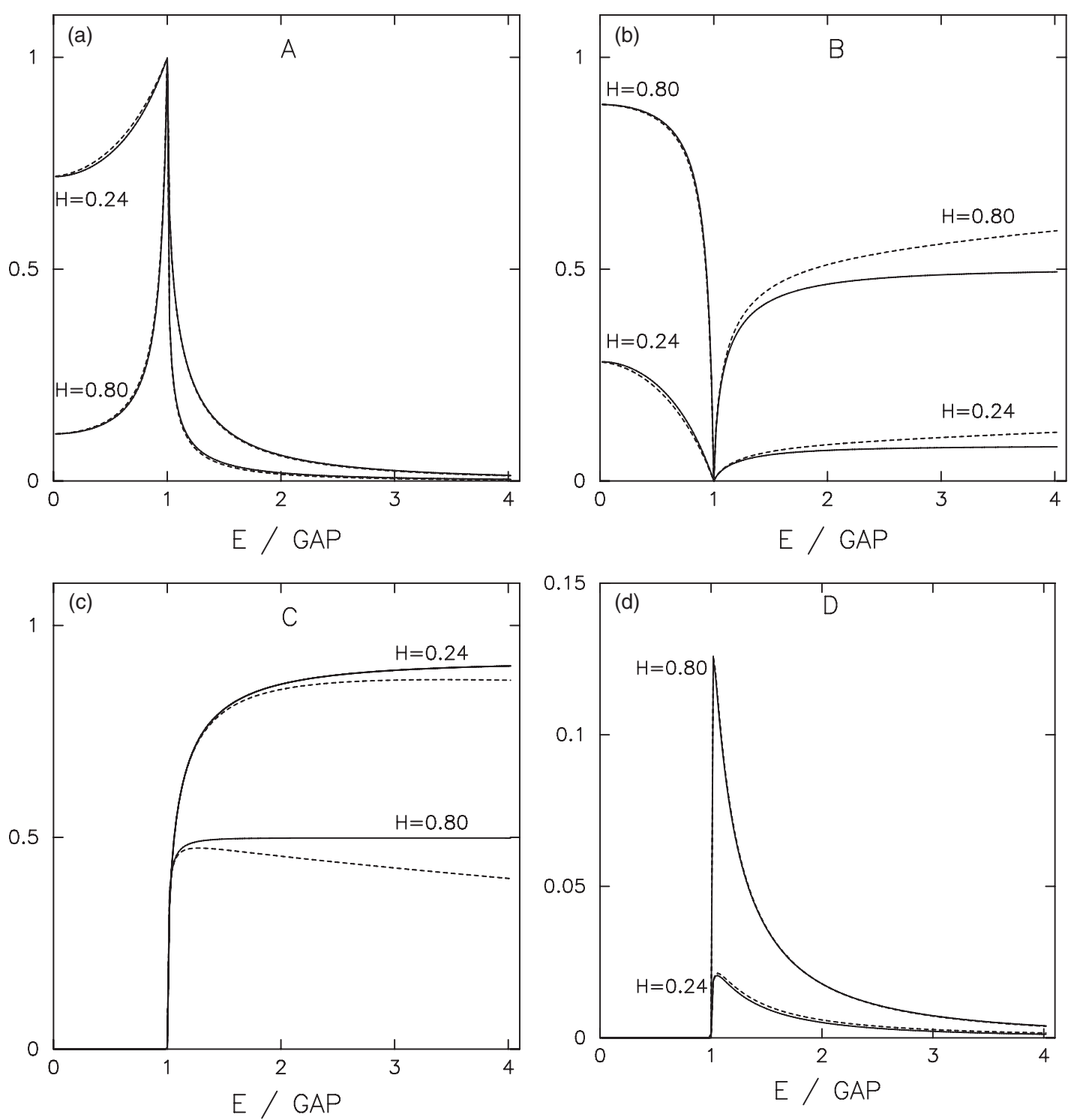

Figure 3. The same reflection and transmission coefficients calculated for the same parameters as in figure 2 and on the same scale, but with the speed of light set equal to $1 / 1000$ th of its actual value to emphasize relativistic effects. The calculations are shown for $H=0.24$ and $H=0.80$. Full lines are the non-relativistic result, dashed lines are the results of the relativistic theory. (a) The probability of Andreev reflection; (b) the probability of normal reflection; (c) the probability of transmission with no band branch crossing; (d) the probability of transmission with a band branch crossing.

and again the boundary conditions lead to a set of equations that are inconsistent and so there is no general solution. However, if we set $I=0$ so there is no incident particle the equations do have a consistent non-trivial solution. One of the coefficients is chosen arbitrarily and the remainder are written in terms of it. We choose to write the other coefficients in terms of $f$,

$$
\begin{array}{cc}
I=c=d=0 & a=\frac{Z\left(v_{1}^{2}-u_{2}^{2}\right)-i u_{2}}{(1+i Z) u_{2}} f \\
b=\frac{v_{1}}{1+i Z} f & e=-\frac{i Z v_{1}}{(1+i Z) u_{2}} f
\end{array}
$$

and if we evaluate the total current density it is

$$
J_{\mathrm{T}}(E)=\frac{2 c^{2} p_{z}}{W+m c^{2}}\left(\left|u_{2}\right|^{2}-\left|v_{1}\right|^{2}\right)|f|^{2}
$$

which is what we would expect. The probability of being in any state has been written in terms of $f$ and the current density is just the usual prefactor multiplied by the probability that the quasiparticle is an electron minus the probability that it is a hole. In this case the model appears to be predicting a spontaneous process that may occur only in the absence of an incident electron. In general though, we find that these processes either do not conserve energy or do not conserve particles. In fact, with no incident electron there is no energy incident upon the interface, so none of these processes can occur except as fluctuations within the uncertainty principle. 
3.3.4. A spin down electron and a spin down hole. In this case the incident particle wavefunction is

$$
\begin{aligned}
& \psi_{n}(z)=I\left(\begin{array}{c}
1 \\
0 \\
\frac{c \hbar k}{W+m c^{2}} \\
0 \\
0 \\
0 \\
0 \\
0
\end{array}\right) \mathrm{e}^{\mathrm{i} p_{z} z / \hbar}+a\left(\begin{array}{c}
0 \\
0 \\
0 \\
0 \\
0 \\
i \\
0 \\
\frac{i c p_{z}}{W+m c^{2}}
\end{array}\right) \mathrm{e}^{\mathrm{i} p_{z}^{+} z / \hbar} \\
& +b\left(\begin{array}{c}
1 \\
0 \\
\frac{c \hbar k}{W+m c^{2}} \\
0 \\
0 \\
0 \\
0
\end{array}\right) \mathrm{e}^{-\mathrm{i} p_{z} z / \hbar} .
\end{aligned}
$$

The boundary conditions soon lead to

$$
\begin{array}{crl}
b=e=f=0 & a=-i \frac{v_{2}}{u_{1}} \\
c=\frac{1-i Z}{u_{1}} & d=\frac{i Z v_{2}}{u_{1}^{2}} .
\end{array}
$$

These results are the same as in the section on a spin up electron and a spin down hole when $b$ has been set equal to zero. This is reassuring, as the results have to be identical in this limit. Interestingly, this limit gives us further insight into that case. These results for $a, b, c$ and $d$ are what the results for the spin up electron and spin down hole reduce to if $\left|u_{1}\right|^{2}=\left|v_{2}\right|^{2}$. One may examine equations (33) and (34) further and we see then that the term in $Z^{2}$ in the definition of $\gamma$ only arises because normal reflection is possible. In general, the $Z^{2}$ term increases the denominator and hence reduces the magnitude of $c$ and $d$. This is reasonable because the fact that electrons may be reflected reduces the number of electrons that tunnel into the superconductor and hence reduces the current density in the superconductor.

\subsection{Scattering at the interface beyond the BTK approximation}

It is clear that if we wish to see any relativistic effects in the $I-$ $V$ curves at a normal/superconductor junction it is necessary to go beyond the BTK theory. We do this in the most minimal way possible. We relax our approximations on the wavevector slightly, but still insist that

$$
k^{+}=q^{+} \quad k^{-}=q^{-} .
$$

We do not insist that either $k^{+}$or $k^{-}$are equal to the Fermi wavevector. This approximation makes the arithmetic considerably more complicated. Defining

$$
Z_{k}^{+}=\frac{\left(W\left(k^{+}\right)+m c^{2}\right) H}{2 \hbar^{2} c^{2} k^{+}} \quad Z_{k}^{-}=\frac{\left(W\left(k^{-}\right)+m c^{2}\right) H}{2 \hbar^{2} c^{2} k^{-}}
$$
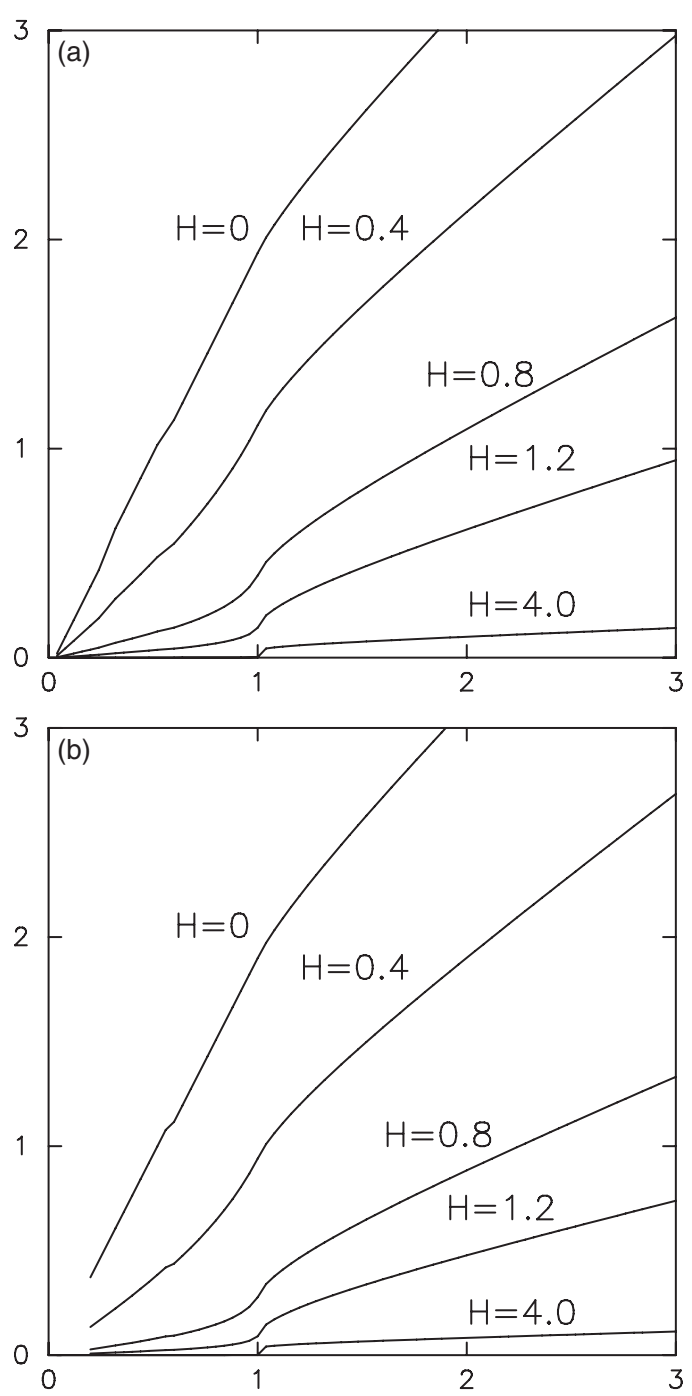

Figure 4. Current (vertical axis) versus potential difference (horizontal axis) curves for a variety of barrier strengths at $T=0$ for the case of a spin up electron and a spin down hole on the normal side of the barrier. The current is calculated using equation (45) with $2 N(0) v_{\mathrm{F}} \Lambda$ set equal to unity. Both the current and potential difference are written in dimensionless units to facilitate comparison with the non-relativistic results [13]. The potential difference is multiplied by the electronic charge (unity in these units) and divided by the energy gap to make it dimensionless $(e V / \Delta)$ and as independent as possible of the parameters of the model. The current is multiplied by the electronic charge and a unit resistance and divided by the energy gap for the same reason $(e I R / \Delta)$.

(a) Relativistic theory with the speed of light set to its actual value. These results are identical to those found within the non-relativistic theory by Blonder [13]. (b) Relativistic theory with the speed of light set equal to $1 / 1000$ th of its actual value to emphasis relativistic effects.

we eventually find

$$
\begin{aligned}
d(E) & =2 Z_{k}^{-}\left(Z_{k}^{-}-Z_{k}^{+}+2 i Z_{k}^{-} Z_{k}^{+}\right) v_{2}(E) \\
& \times\left\{4\left(Z_{k}^{+} Z_{k}^{-}\left(1+i Z_{k}^{+}\right)\left(1-i Z_{k}^{-}\right)\right) u_{1}^{2}(E)\right. \\
& \left.+\left(Z_{k}^{-}-Z_{k}^{+}+2 i Z_{k}^{-} Z_{k}^{+}\right)^{2} v_{2}^{2}(E)\right\}^{-1}
\end{aligned}
$$

and the remaining coefficients can be written in terms of this as 

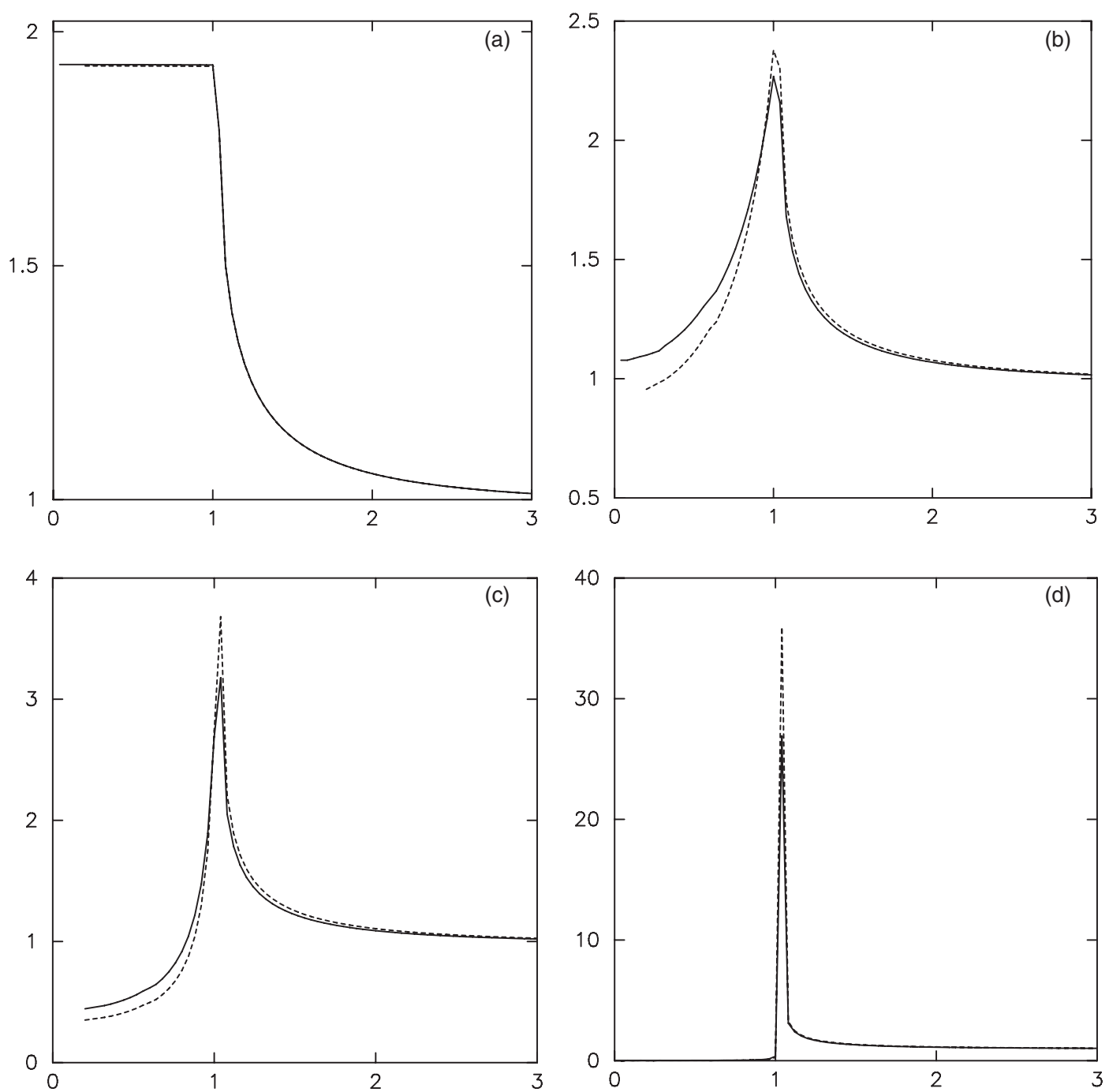

Figure 5. Differential conductance $R_{n} \mathrm{~d} I / \mathrm{d} V$ where $R_{n}$ is a unit resistance included to make the differential conductance dimensionless and directly comparable with Blonder et al [13] as a function of energy in units of the energy gap. This is for the case of a spin up electron and a spin down hole on the normal side of the barrier. The results are plotted for a variety of barrier strengths. In each graph the full line is the true relativistic result and the dashed line is the result with the speed of light set equal to $1 / 1000$ th of its value: (a) $H=0$; (b) $H=0.4$;

(c) $H=0.8$; (d) $H=4.0$.

$$
\begin{gathered}
c(E)=\frac{2 Z_{k}^{+}\left(1-i Z_{k}^{-}\right) u_{1}(E)}{\left(Z_{k}^{-}-Z_{k}^{+}+2 i Z_{k}^{-} Z_{k}^{+}\right)} d \\
b(E)=c(E) u_{1}+d(e) v_{2}-1 \\
a(E)=c(E) v_{2}+d(E) u_{1} .
\end{gathered}
$$

Equations (43) and (44) reduce to the BTK limit if we set $Z_{k}^{-}=$ $Z_{k}^{+}$. The reflection and transmission coefficients can now be calculated using equations (43) and (44) in equations (26). The results are not presented separately here because they are very similar indeed to those shown in figure 2 . For reasonable values of the parameters the difference between the relativistic and non-relativistic theories is indistinguishable on this scale. Generally, we find that even in the most advantageous case the difference between the two approaches is about one part in $10^{4}$ and so completely negligible.

It is of interest to see how relativity affects these coefficients, and we can do this artificially either by reducing the speed of light or increasing the energy gap until relativistic effects become appreciable. In figure 3 we show the transmission coefficients calculated for the same gap as in figure 2 , but with the speed of light set equal to $1 / 100$ th of its actual value. These are displayed for $H=0.24$ and $H=0.80$ only because relativistic effects are less visible in the more extreme values of $H$.

The qualitative reasons for the observed effects of relativity are simple to understand. With $c$ set sufficiently low, the numerators of equations (42) are no longer so dominated by the $m c^{2}$ and $Z$ becomes a much more sensitive function of $k$ and hence of $E$. In figure $2 Z(\equiv H)$ is constant; in figure $3 H$ remains constant; but $Z$ increases substantially as we move from left to right on each figure. Therefore the coefficients are characteristic of a higher $Z$ on the right of the figure than they are on the left of the figure. A change in curvature of $A$ and $B$ at low energy at $H=0.25$ is just visible in these figures. As relativistic effects become more important, energy is no longer 
quadratically dependent on momentum and this leads to the observed effect.

\subsection{The normal/superconductor boundary at finite voltage}

Our discussion of the calculation of current-voltage and differential conductance curves as well as excess current is identical to that of BTK [13]. To minimize repetition we refer the reader to that paper and only mention here the essential points.

If a potential difference $V$ is applied across the nor$\mathrm{mal} / \mathrm{superconductor}$ boundary non-equilibrium quasiparticle populations will be generated in general, which can be determined by solving the Boltzmann equation self-consistently. If we make the assumption that all incoming particles are described by a standard Fermi-Dirac distribution function $f_{0}(E)$, the problem is greatly simplified and the expression for the current is derived by Blonder et al [13] as

$$
\begin{aligned}
I_{N S} & =2 N(0) e v_{\mathrm{F}} \Lambda \\
& \times \int\left[f_{0}(E-e V)-f_{0}(E)\right][1+A(E)-B(E)] \mathrm{d} E
\end{aligned}
$$

where $\Lambda$ is the area of the junction, $N(0)$ is the density of states per spin direction at the Fermi energy and $v_{\mathrm{F}}$ is the Fermi velocity. In the absence of Andreev scattering this reduces to an Ohm's law form,

$$
I_{N N}=\frac{2 N(0) e^{2} v_{\mathrm{F}} \Lambda}{1+Z^{2}} V=\frac{V}{R_{N}}
$$

which defines the normal metal ohmic resistance. In figure 4 we show some $I-V$ curves calculated using equation (45) for a range of values of the parameters. Figure 4(a) is the usual relativistic result and represents identical results to those shown in figure 6 of Blonder et al [13], although the vertical scale in their picture differs from ours. In figure 4(b) we display the same quantities with the speed of light set equal to 1/1000th of its actual value. Again we can see the effect of relativity easily. For a given barrier and potential difference the current is lower in figure 4(b) than 4(a). This is because, although $H$ is the same in both diagrams in figure $4(\mathrm{~b}), Z$ increases more rapidly with energy and so when compared with figure 4(a) the curves in figure 4(b) tend to higher values of $Z$. From these curves it is trivial to calculate the differential conductances $\mathrm{d} I / \mathrm{d} V$ and these are shown in figure 5 for a representative set of barrier strengths. Again, the full lines have the speed of light as its actual value and the results are essentially identical to figure 7 of Blonder et al. As one might expect from the previous discussion, the effect of relativity is shown to make the peak in this curve sharper, i.e. to make it more like the nonrelativistic result for higher $Z$.

In some junctions, where Andreev scattering is significant, the $I-V$ curves are still approximately linear, but do not extrapolate to zero. At the point where such curves intersect the $I$-axis we can define an 'excess current'. This can be defined as

$$
\begin{aligned}
I_{\mathrm{exc}} & =I_{N S}-\left.I_{N N}\right|_{e V \gg \Delta} \\
& =\int_{0}^{\infty} \frac{1+Z^{2}}{e R_{N}}\left[A(E)-B(E)+\frac{Z^{2}}{1+Z^{2}}\right] \mathrm{d} E .
\end{aligned}
$$

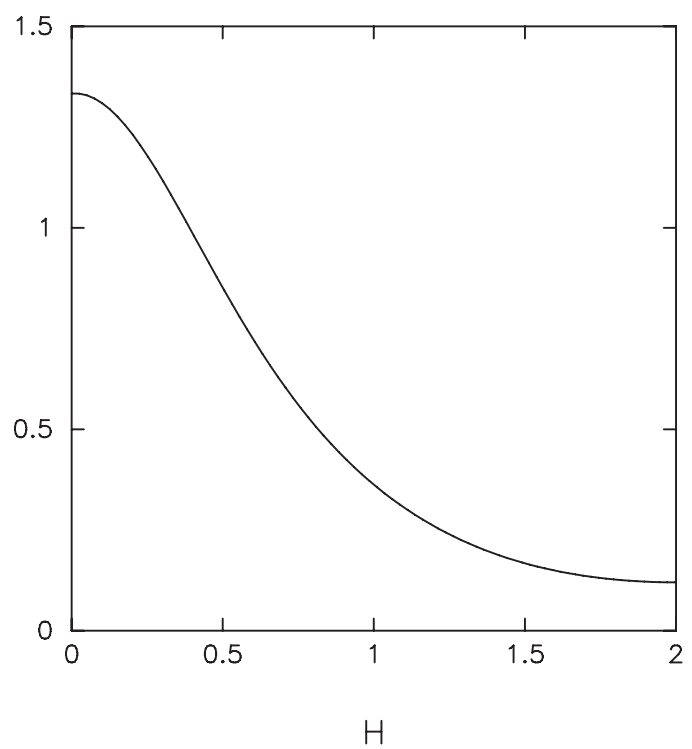

Figure 6. The excess current as a function of barrier strength $H$ calculated using equation (47) and relativistic values of the parameters calculated from equations (26) and (43) and (44). The result is for the case of a spin up electron and a spin down hole on the normal side of the barrier and is independent of the value used for the speed of light. Obviously therefore this result is identical to the non-relativistic value.

In figure 6, we show the excess current calculated using equation (47), and it is essentially identical to the nonrelativistic result. Relativity seems to have no effect on this quantity. Although the integrand differs for different values of the speed of light (especially $B(E)$ as exemplified in figure $3(\mathrm{~b}))$, the integral is independent of it.

\section{Conclusions}

The original BTK paper [13] derived and interpreted a simple model of a normal metal/superconductor junction that was able to explain several experimental observations, including characteristic $I-V$ curves for junctions with an arbitrary barrier strength, excess currents, current conversion and charge imbalance. We have derived a relativistic generalization of the BTK theory of transport through normal/superconducting contacts. This has been shown to reduce to the non-relativistic theory correctly as $c \rightarrow \infty$. We have observed that if we make the same approximations as the BTK theory there are rigorously no relativistic effects. However, we have been able to use this formalism to gain further insight into the scattering by demonstrating that the $Z^{2}$ term in the definition of $\gamma$ arises from the fact that normal reflection is allowed. If the BTK approximations are not made, relativistic effects are found to be very small in systems with realistic values of the parameters. As we have shown, this tells us that the relativistic theory describes all the physics that the non-relativistic theory describes. With artificial values of the parameters we have been able to show that the effect of relativity is to make the effective barrier strength greater and hence to enhance the scattering. We reaffirm the statement made in the introduction that the main results of interest in this work are the formalism itself and the 
insight gained into the effect of relativity on junctions rather than any deeper interpretation of experiment.

\section{Appendix A. Conservation laws}

Here we consider the equation of continuity obeyed by the solutions of the Bogoliubov-de Gennes equations. Using standard methods [25], we can derive an equation of the form

$$
\frac{\partial \rho(z, t)}{\partial t}=-\frac{\mathrm{d}}{\mathrm{d} z} j(z, t) .
$$

where the probability density and current are given by

$$
\begin{gathered}
\rho(z, t)=|u(z, t)|^{2}+|v(z, t)|^{2} \\
j(z, t)=u^{\dagger}(z, t) c \alpha_{z} u(z, t)+v^{\dagger}(z, t) c \alpha_{z} v(z, t)
\end{gathered}
$$

where the dagger represents the hermitian conjugate matrix. Interestingly, the current has a positive sign in it while the non-relativistic theory has a negative sign. One would expect the hole current to be in the opposite direction to the electron current, so this looks incorrect. However, the $\alpha_{z}$ matrix contains negatives and combines with the signs in the eigenfunctions to always yield the hole current in the opposite direction to the electron current in the superconductor.

We can also derive a conservation law for quasiparticle charge. An electron has a charge $+e$ and a hole has a charge $-e$ and so the quasiparticle charge density is

$$
Q=e\left(|u(z, t)|^{2}-|v(z, t)|^{2}\right) .
$$

With this definition it is straightforward to derive the conservation law of the form

$$
\frac{\partial Q}{\partial t}=-\nabla J_{Q}(z, t)-\nabla J_{S}(z, t) .
$$

The first term here is written in terms of the quasiparticle current

$$
J_{Q}(z, t)=e c \operatorname{Re}\left(u^{\dagger}(z, t) \alpha_{z} u(z, t)-v^{\dagger}(z, t) \alpha_{z} v(z, t)\right) .
$$

The second term on the right here is a source/sink term that represents the supercurrent.

$$
J_{S}(z, t)=\frac{4 e \Delta}{\hbar} \int \operatorname{Im}\left(u^{\dagger}(z, t) \eta v(z, t)\right) \mathrm{d} z .
$$

\section{Appendix B. A simple model}

In this appendix we examine the simplest model possible that may capture the quasiparticle current being converted into the condensate current. This model was also examined nonrelativistically by Blonder et al [13]. We consider the case $H=0$ where there is no barrier between the normal metal and the superconductor. We will also look at the case when $E<\Delta$. In this case $b=d=0$. We will look at the case of an incident spin up particle, and we also know from earlier that $e=f=0$ in this case. Furthermore, $a$ and $c$ simplify to

$$
a=\frac{i v_{2}}{u_{1}} \quad c=\frac{1}{u_{1}} .
$$

Now, on the normal side of the barrier there is no reflected wave and $a a^{*}=1$ so the electron is entirely Andreev reflected, so the wavefunction is

$$
\psi_{n}(z)=\left(\begin{array}{c}
1 \\
0 \\
\frac{c \hbar k}{W+m c^{2}} \\
0 \\
0 \\
0 \\
0 \\
0
\end{array}\right) \mathrm{e}^{\mathrm{i} p_{z}^{+} z / \hbar}+a\left(\begin{array}{c}
0 \\
0 \\
0 \\
0 \\
0 \\
i \\
0 \\
\frac{i c p_{z}}{W+m c^{2}}
\end{array}\right) \mathrm{e}^{\mathrm{i} p_{z}^{-} z / \hbar}
$$

and on the superconducting side it is

$$
\psi(\mathbf{r}, t)=c\left(\begin{array}{c}
u_{1} \\
0 \\
\frac{c p_{z}}{W+m c^{2}} u_{1} \\
0 \\
0 \\
v_{2} \\
0 \\
\frac{c p_{z}}{W+m c^{2}} v_{2}
\end{array}\right) \mathrm{e}^{\left(\mathrm{i} p_{z}^{+} z-E t\right) / \hbar}
$$

Now we have a steady state with a current passing through it. The current can be calculated anywhere and so is independent of $z$ and $t$. Let us start by evaluating it on the normal side of the interface. Using equation (56) in (53) we find

$$
J_{Q}=\frac{4 e c^{2} p_{z}}{W+m c^{2}} .
$$

This can be checked by taking the non-relativistic limit and comparing with Blonder et al [13], and in perfect agreement with them we find

$$
\lim _{c \rightarrow \infty} \frac{4 e c^{2} p_{z}}{W+m c^{2}}=2 e v_{\mathrm{F}}
$$

if $p_{z}$ is taken to be the Fermi momentum. $v_{\mathrm{F}}$ is the Fermi velocity. Next we evaluate $J_{Q}$ within the superconductor using the wavefunction (57). We need to know $p^{+}$and this is given by equation (19). Approximating

$$
\frac{\hbar^{2} k^{+^{2}}}{2 m}=W-m c^{2}
$$

and remembering that we are considering the case when $E<$ $\Delta$ we get

$$
k^{+}=k_{\mathrm{F}}+\frac{1}{\hbar v_{\mathrm{F}}}\left(E^{2}-\Delta^{2}\right)^{1 / 2}=k_{\mathrm{F}}+\frac{i \xi}{\hbar v_{\mathrm{F}}}
$$

where

$$
\xi=\left(\Delta^{2}-E^{2}\right)^{1 / 2}
$$

$k^{+}$is imaginary and so the wavefunction decreases exponentially. This is to be expected; it is clear from (15) that there is 
no wavelike solution in this energy regime. The wavefunction becomes

$$
\psi(\mathbf{r}, t)=c\left(\begin{array}{c}
u_{1} \\
0 \\
\frac{c p_{z}}{W+m c^{2}} u_{1} \\
0 \\
0 \\
v_{2} \\
0 \\
\frac{c p_{z}}{W+m c^{2}} v_{2}
\end{array}\right) \mathrm{e}^{\mathrm{i}\left(k_{\mathrm{F}}+i \xi / \hbar v_{\mathrm{F}}\right) z}
$$

and calculating $J_{Q}$ using equation (53) we find

$$
J_{Q}=\frac{2 e c^{2} p_{z}}{W+m c^{2}}\left(\frac{\left|u_{1}\right|^{2}+\left|v_{2}\right|^{2}}{\left|U_{1}\right|^{2}}\right) \exp \left(\frac{-2 \xi z}{\hbar v_{\mathrm{F}}}\right) .
$$

When $E<\Delta \quad\left|u_{1}\right|^{2}=\left|v_{2}\right|^{2}$ and so

$$
J_{Q}=\frac{4 e c^{2} p_{z}}{W+m c^{2}} \exp \left(\frac{-2 \xi z}{\hbar v_{\mathrm{F}}}\right)
$$

Clearly at $z=0$ this is identical to equation (58) but as we get deeper into the superconductor the current decreases exponentially. This has to be taken care of via the source/sink term in (52). We now evaluate this directly using equation (63) in the source term.

It is easy to show that

$$
u^{\dagger}(z, t) \eta v(z, t)=\frac{v_{2}}{u_{1}} \frac{2 W}{W+m c^{2}} \exp \left(-\frac{2 \xi z}{\hbar v_{\mathrm{F}}}\right)
$$

and a little further algebra shows that

$$
J_{s}(z)=\frac{4 e p_{z} c^{2}}{W+m c^{2}}\left(1-\exp \left(-\frac{2 \xi z}{\hbar v_{\mathrm{F}}}\right)\right) .
$$

Clearly, adding this to (65) we get back equation (58) as we must. This term represents the supercurrent, which increases to its asymptotic value as $z \rightarrow \infty$.

\section{References}

[1] Kammerlingh Onnes H 1911 Leiden Commun. 122b 124

[2] Cooper L N 1956 Phys. Rev. 1041189

[3] Bardeen J, Cooper L N and Schreiffer J R 1957 Phys. Rev. 1081175

[4] de Gennes P 1966 Superconductivity in Metals and Alloys (New York: Benjamin)

[5] Tinkham M 1996 Introduction to Superconductivity (New York: Dover)

[6] Annett J F 2004 Superconductivity, Superfluids and Condensates (Oxford: Oxford University Press)

[7] Capelle K and Gross E K U 1995 Phys. Lett. A 198261

[8] Giaever I 1960 Phys. Rev. Lett. 5147

[9] Goldberger M L and Watson K M 1964 Collision Theory (New York: Wiley)

[10] Cuevas J C, Martin-Rodero A and Levy Yeyati A 1996 Phys. Rev. B $\mathbf{5 4} 736$

[11] Mahan G D 1990 Many Particle Physics (New York: Plenum)

[12] Arnold G B 1985 J. Low Temp. Phys. 59143 Arnold G B 1987 J. Low Temp. Phys. 681

[13] Blonder G E, Tinkham M and Klapwijk T M 1982 Phys. Rev. B 254515

[14] Lambert C J 1991 J. Phys.: Condens. Matter 36579

[15] Kupka M 1990 J. Phys.: Condens. Matter 210599

[16] Mortensen N A, Flensberg K and Jauho A P 1999 Phys. Rev. B 5910176

[17] Kupka M 1997 Physica C 281 91-106

[18] Soulen R J, Byers J M, Osofsky M S, Nadgorny B, Ambrose T, Cheng S F, Broussard P R, Tanaka C T, Nowak J, Moodera J S, Barry A and Coey J M D 1998 Science 282 85-8

[19] Blonder G E and Tinkham M 1983 Phys. Rev. B 27112

[20] Kastalsky A, Kleinsasser A W, Greene L H, Bhat R, Milliken F P and Harbison J P 1991 Phys. Rev. lett. 673026

[21] Santhanam P, Chi C C, Wind S J, Brady M J and Buchignano J J 1991 Phys. Rev. lett. 662254

[22] Raychaudhuri P, Jaiswal-Nagar D, Sheet G, Ramakrishnan S and Takeya H 2004 Phys. Rev. Lett. 93156802

[23] Bogoliubov N N 1958 Nuovo Cimento 7794

[24] Valatin J G 1958 Nuovo Cimento 7843

[25] Strange P 1998 Relativistic Quantum Mechanics (Cambridge: Cambridge University Press) chapter 4

[26] Fairbairn W M, Glasser M L and Steslicka M 1973 Surf. Sci. 36462 